\title{
Conectividad, hipermedialidad y multimodalidad: de la cultura digital al espacio escolar ${ }^{1}$
}

\section{Connectivity, Hypermediality and Multimodality: from Digital Culture to School Environment}

\section{Antonio Quintana Ramírez²}

Citation/ Para citar este Artículo: Quintana Ramírez, A. (2020). Conectividad, hipermedialidad y multimodalidad: de la cultura digital al espacio escolar. Colomb. Appl. Linguistic. J., 22(2), pp. 207-220.

Received: 2-Jun.-2020 / Accepted: 19-Dec.-2020

DOI: https://doi.org/10.14483/22487085.16467

\section{Resumen}

Este artículo da cuenta de los resultados de la tesis doctoral: "Hipertextualidad y conectividad: alternativas de la cultura digital para la configuración de ambientes educativos", que tuvo como objetivo identificar y describir los elementos que caracterizan las prácticas educativas en ambientes en los que se favorece la conectividad, la hipermedialidad y la mutimodalidad, y que, junto con la cibercultura, se erigieron como categorías teóricas. La investigación consistió en un estudio microtecnográfico desarrollado en una Institución Educativa Distrital (IED) con un grupo de 30 estudiantes de grado quinto, en aulas dotadas con tabletas y plena conectividad. Dentro de los hallazgos y conclusiones del estudio se destacan: 1) el surgimiento del espacio de interacción como un hipermedio en sí mismo; 2) una relación entre procesos de juego y aprendizaje; 3) la aparición de nuevas dinámicas de atención distribuida; 4) la diferenciación simbólica entre el laboratorio y el aula de clase; 5) el reconocimiento de que las propuestas pedagógicas no son tan nuevas como las tecnologías digitales; 6) las tabletas no favorecen la escritura hipermedial, pero sí la multimodal y la hibridación entre lo digital y lo análogo.

Palabras clave: cibercultura, conectividad, hipermedia, multimodalidad, tabletas y educación

\begin{abstract}
This paper presents the main results achieved in the doctoral dissertation: "Hypertextuality and connectivity: alternatives of digital culture for the configuration of educative environments". It focused on identify and describe the elements that characterize educational practices in educational environments in which connectivity, hypermediality and multimodality, along with the context of cyberculture, are promoted as central theoretical categories. The research corresponds to a micro-technographic study developed in IED, with a group of 30 students of 5 th grade, in a specialized classroom equipped with tablets and full connectivity. The most important results and insights of the study are: 1) the emergence of the space of interaction as hypermedia itself; 2) a relationship between play and learning processes; 3) new dynamics of distributed attention; 4) symbolic distinction between the laboratory and the classroom; 5) to realize that pedagogical proposals are not as new as digital technologies; 6) due to its architecture, tablets do not help hypermedial writing; but multimodal writing and hybridization between digital and analogic technologies.
\end{abstract}

Keywords: cyberculture, connectivity, hypermedia, multimodality, tablets and education

1 Este artículo es resultado de la investigación de la tesis doctoral del autor.

2 Docente titular de la Universidad Distrital Francisco José de Caldas, candidato a Doctor del Doctorado Interinstitucional en Educación de la misma institución. ORCID (D): https://orcid.org/0000-0002-2912-6542. aquintana@udistrital.edu.co 


\section{Introducción}

El objetivo principal de esta investigación fue realizar una descripción interpretativa de las prácticas educativas de estudiantes y docentes en ambientes educativos con plena conectividad, acceso individual a tabletas y uso del potencial de interactividad e hipermedialidad. La pregunta central del estudio fue: ćqué elementos permiten describir las prácticas educativas de los sujetos que participan en entornos hipermedializados e interconectados?

El estudio se realizó en el marco del proyecto piloto Smart School con apoyo de la Secretaría de Educación Distrital (SED). En este proyecto se trabajó con grupos de estudiantes de distintas localidades de la ciudad de Bogotá y, en particular, esta investigación centró la atención en un grupo natural de 30 estudiantes de grado $5 .^{\circ}$ de una IED.

El escenario de la indagación tuvo dos espacios: el laboratorio del Centro Ático y el colegio mismo. El espacio físico de este último contó con la dotación de 30 tabletas y la conectividad que proveyó la SED a través de la Red Integrada de participación educativa -REDP-.

\section{Marco teórico}

Este trabajo considera la cibercultura como elemento de fundamentación teórica: desde esta perspectiva cultural se asumió el estudio de las potencialidades educativas de estas tecnologías. De igual manera, reconoce la hipertextualidad y la multimodalidad como elementos caracterizadores de estas nuevas maneras de narrar-se y de acceder al mundo de la información. Por último, incluye la conectividad, entendida como la condición que hace posibles las formas de interacción social con los contenidos de la red.

\section{Sobre la cibercultura}

Según Lévy (2007), la cibercultura corresponde al conjunto de sistemas culturales que ha emergido en estrecha relación con las tecnologías digitales y ha configurado un nuevo espacio de interacción social: el ciberespacio ${ }^{3}$. Desde una perspectiva antropológica, Escobar (2005) hace énfasis en el poder transformador de las reconstrucciones culturales en las que las nuevas tecnologías están basadas y a las que ayudan a dar forma.

Ese nuevo mundo cultural es el producto de la interacción y mutua afectación entre las prácticas, usos, apropiaciones y modificaciones, mediadas por los instrumentos tecnológicos, ${ }^{4}$ con el mundo de representación y expresión simbólica de los individuos en entornos de socialización, pues

\begin{abstract}
una cultura comprende no solo capacidades, actividades y realizaciones de carácter simbólico (tales como representaciones e interpretaciones simbólicas, discursivas, artísticas, teóricas, cosmovisivas, colorativas, etc., es decir, la cultura en su acepción más restringida), sino también técnicas y artefactos materiales [...], formas organizativas de interacción social, económica y política. (Medina, 2003, p. 44)
\end{abstract}

Estos entornos simbólicos se caracterizan por la emergencia y el impacto contemporáneo de los nuevos medios -los digitales, los de la red- (Igarza, 2008) que se traslapan, complementan y, por momentos, se interpelan con los medios masivos.

En este contexto, el lenguaje audiovisual se ha superpuesto a la oralidad y a la palabra escrita gracias a la proliferación de las pantallas en las que la imagen es primacía. Para su comprensión, estas ameritan "una antropología cultural de las

3 William Gibson (1997) acuñó el término en su novela Neuromante, publicada inicialmente en 1984. Ciberespacio es una voz que hace parte del lenguaje emergente con los desarrollos de las tecnologías digitales. Echeverría (2001) afirma que las tecnologías de la información y de la comunicación (TIC) posibilitan la construcción de un nuevo espacio-tiempo social: el ciberespacio. Lévy (2004) hace un llamado a la concepción propuesta por Dyson et. al (1994) en la que proponen el ciberespacio como "la tierra del conocimiento" (p. 297).

4 La referencia a instrumentos producidos por la tecnología incluye tanto al conjunto de artefactos con los que interactuamos cotidianamente como al sinnúmero de procesos y a los complejos sistemas producidos por las tecnologías. Estos dos últimos tienden a ser más transparentes, menos evidentes, pero no por ello menos relevantes. 
superficies, capaz de dar cuenta de las mediaciones técnicas, semánticas y estéticas que organizan la producción y la reproducción de los sujetos sociales" (Piscitelli, 2002, p. 21). En la cultura digital, la escuela contemporánea ha de asumir retos para la constitución de nuevas narrativas en las que el poder letrado -afincado en su capacidad para institucionalizarse (Rama, 1998, p. 35) - empieza a ceder espacio a nuevas maneras de narrar que hacen posible la alternativa de una escuela hipertextualizada. En este tipo de escuela, la imagen, la fragmentación, la multilinealidad, la participación, la producción colectiva, los aprendizajes colaborativos en red y, en general, las nuevas interacciones toman su lugar, como se podrá evidenciar más adelante. Según Bisama (2002), se trata del desplazamiento de la ciudad letrada hacia una hipertextualizada que la reemplaza "en la medida que propone modalidades nuevas de intercambio cultural, además de hibridar las existentes" (s. p.).

\section{Hipermedialidad y lectoescritura}

Desde los primeros trabajos pedagógicos de los sistemas hipermediales (Scardamalia y Bereiter, cómo se citó en de Corte, 1996; Palumbo y Prater, 1993; Swan y Meskill, 1996; Yang, 1996, entre otros), se evidenció el favorecimiento del aprendizaje de la escritura debido a una mayor motivación de parte de los estudiantes, catapultada -entre otros elementos- por el apoyo al desarrollo del aprendizaje autónomo y cooperativo, el uso de representaciones no lineales del conocimiento, las posibilidades de aprendizaje y para distintos estilos, y el trabajo de producción escritural colectiva. Estudios en el contexto nacional (Clavijo y Quintana, 2004; González y León, 2002; Henao, 2002; Henao et. al, 2004; Quintana et. al, 1998) mostraron que el enfoque hipermedia, que permite múltiples historias usando un guion gráfico $^{5}$ de nodos y enlaces, es más flexible que el enfoque lineal y, por lo tanto, favorece aspectos como la coherencia y la cohesión local y global. Además, el uso de mapas o esquemas permite diseñar las rutas y estructuras del hipertexto, y el

5 Estos guiones gráficos sitúan la realización de mapas como elementos clave de la composición hipermedial (Pohl y Purgathofer, 2004). apoyo entre compañeros influye positivamente en la interdependencia positiva ${ }^{6}$.

Sobre la comprensión lectora en hipermedios, Hamdan et. al (2017) y Salmerón et. al (2017) mostraron que el procesamiento profundo de la información, la presencia de textos en fragmentos breves y puntuales, así como la inclusión de imágenes, tablas, diagramas, materiales de audio y videos -juntos o articulados con el texto- favorecen la comprensión lectora.

En el campo de la literatura hipermedial, "la era digital no solo está ampliando los medios y métodos de cómo leemos y escribimos, sino también la noción de lo "literario"' (Gorman, 2017, p. 1). En este contexto se encuentran múltiples propuestas de narrativa hipertextual o hyper-literature ${ }^{7}$, que, tal como lo expone Pope (2017), han superado muchas de las barreras implícitas para los lectores de estas narrativas, aunque tales impedimentos no hayan desaparecido totalmente: "A pesar de los grandes avances técnicos $y$, aunque algunos ejemplos recientes están funcionando bien comercialmente, la narrativa digital interactiva sigue siendo en gran parte desconocida para el público lector general" (p. 1).

\section{Sobre la multimodalidad}

Según Kress (2009), la multimodalidad es una teoría que estudia los diferentes modos de comunicación y expresión que tienen características fuertemente vinculadas a las tecnologías digitales. Es importante destacar que esta teoría se inscribe

6 "La interdependencia positiva [es] la creencia de cada individuo de que hay un valor en el aprendizaje con otros alumnos y que tanto la capacidad individual como los productos de trabajo se incrementará como resultado de la colaboración" (Johnson y Johnson, 1994, como se citó en Liu et. al, 2011, p. 1545).

7 Algunos ejemplos son: Inanimate Alice, narrativa multimodal de The Bradfield Company (2006); Pry, obra de Gorman y Cannizzaro (2013), explora las posibilidades del juego y los gestos hápticos; The Ghosts of the Yamaraja de Saunders (2013) integra el celular del lector-protagonista; Underbelly, de Wilks (2010), ofrece un ambiente de exploración de sonidos y voces que se traslapan al recorrer un oscuro espacio del inframundo; Gabriella infinita, Golpe de gracia y Memorias y caminos de Rodríguez (2011) son obras que privilegian la exploración y participación interactiva del lector de fragmentos, sin articulación explícita. 
en el enfoque de la semiótica social, que pretende ir más allá del análisis lingüístico del signo y se instala en el lugar de las prácticas sociales, en la medida en que "la génesis de los signos radica en las acciones sociales" (p. 54).

El énfasis en el análisis de las acciones sociales (comunicativas, expresivas y de representación) responde al reconocimiento que hace la teoría semiótica social de la creación o producción activa de signos en las interacciones sociales. "Los signos se hacen, más que se utilizan", idea que distingue este enfoque, pues diferencia "la teoría semiótica social de otras formas de semiótica" (Kress, 2009, p. 54).

La multimodalidad reconoce que la gente se comunica en una variedad de formas, y que para entender completamente a alguien los muchos modos utilizados para la comunicación deben ser observados y reconocidos (Jewitt y Kress, 2003; Kress, 2000). En este sentido, Dussel y Gutiérrez (2006) destacan que la imagen "no es un artefacto puramente visual, puramente icónico, ni un fenómeno físico, sino que es la práctica social material que produce una cierta imagen y que la inscribe en un marco social particular" (p. 280).

Kress et. al (2000) enfatizan que hoy existe una caracterización de lo multimodal en la que hay primacía de la imagen sobre la escritura y un dominio de la pantalla sobre el libro: "lo visual es mucho más prominente como forma de comunicación de lo que fue durante tantos siglos [...]. El cambio está produciendo textos que son fuertemente multimodales" (p. 373).

Este cambio ha generado un descentramiento de la textualidad hacia la multimodalidad que no significa el fin de la escritura, mas sí un desplazamiento de la escritura a la visualidad -o quizás sea más preciso decir: hacia la escritura con imágenes-. Según Kress (2003), el extenso uso de la imagen va aparejado a cambios importantes en la reestructuración del poder, ya que la posibilidad de acceso, la producción y el consecuente cambio de la relación "uno a muchos" por la de "muchos a muchos" descentran la relación del poder frente a la producción y la difusión.
Farías y Araya (2014) exponen la pertinencia del reconocimiento de la cultura multimodal para los aprendizajes y que esta se ubique más allá de lo puramente lingüístico:

Si la educación se trata de aprendizaje y si
creamos significados en varios modos de
manera tal que nuestro compromiso con el
mundo es siempre multimodal, entonces parece
simplemente esencial que la institución encargada
de los aprendizajes esté no solo basada en el
aprendizaje de la lengua sino en cómo la cultura
que se le presenta a los niños en la escuela está
representada y cómo los jóvenes hacen sentido
de ella. Eso no se puede hacer solo por medio
de una pedagogía basada en lo lingüístico. (p. 96)

En el contexto de este trabajo, la mirada de lo multimodal se circunscribe a la descripción de sus usos por estudiantes y profesores. No se pretendió, por tanto, hacer un análisis semiótico de las producciones y usos multimodales, mas sí describirlos e interpretarlos a la luz de sus prácticas educativas.

\section{Conectividad y aprendizaje}

Se destacan dos propuestas: por un lado, la de Downes (2012) y Siemens (2004), quienes llevaron a cabo una experiencia "alternativa" denominada "Connectivism and Connective Knowledge", y, por el otro, el Proyecto Facebook, liderado por Piscitelli et. al (2010). En estas dos experiencias la conectividad se erige como el elemento fundamental para la generación de condiciones que favorecen la ruptura de dispositivos de clase centrados en el monopolio del saber en manos del docente, ya que abre las posibilidades de construcción individual y colectiva a partir de la interacción por vía de la interconexión.

Siemens (2004) ha planteado una apuesta conceptual que denomina conectivismo, retomada por Downes (2019) como una teoría del conocimiento y del aprendizaje para la era digital que parte de la consideración de que "además de ofrecer una explicación del aprendizaje en estas redes [...] ofrece una metodología para tal aprendizaje" (p. 116). Esta idea coincide con el planteamiento de Gardner (1994) en la medida en que la inteligencia se interconecta con recursos externos al cerebro humano -libros, 
accesos a la red, a memorias externas, a expertos-. El planteamiento de Siemens (2004) se apoya, en primer lugar, en la perspectiva del caos que,

\begin{abstract}
a diferencia del constructivismo, el cual establece que los aprendices tratan de desarrollar comprensión a través de tareas que generan significado, el caos señala que el significado existe, y que el reto del aprendiz es reconocer los patrones que parecen estar escondidos. (p. 5)

Rueda (2003) coincide y plantea que

el conectivismo es la integración de principios explorados por las teorías de caos, redes, complejidad y autoorganización. El aprendizaje es un proceso que ocurre al interior de ambientes difusos de elementos centrales cambiantes -que no están por completo bajo control del individuo-. (p. 6)
\end{abstract}

En esta perspectiva, los procesos de exploración de los sujetos con su entorno toman relevancia como vía para los aprendizajes.

\section{La metodología}

Este estudio fue de tipo cualitativo, de carácter descriptivo-interpretativo (Polit y Hungler, 2000), con un diseño metodológico de enfoque etnográfico (Hine, 2004) y en el marco de los estudios de tipo microtecnográficos. Se desarrolló a partir de la observación participante que "implica tener en cuenta la existencia del observador, su subjetividad y reciprocidad en el acto de observar" (Sampieri et. al, 2010, p. 600).

La observación se realizó con un grupo de 30 estudiantes de grado $5^{\circ}$ de la IED durante siete meses. Se trató de un total de 19 sesiones alternadas entre el laboratorio de la universidad y el salón de tabletas de la IED. Se tomaron un total de 21 diarios de campo ${ }^{8}$, videos de las sesiones y los productos

8 La validez interna del estudio se obtuvo mediante triangulación entre los documentos primarios de dos investigadores. Adicionalmente se realizó triangulación con otros instrumentos de recolección de la información: videos, grabaciones de audio y los productos de los estudiantes. de los estudiantes. Toda la información (49 códigos y un total de 1375 citas) fue procesada empleando el programa AtlasTi.

El proceso de elaboración del corpus de datos se fundamentó en el registro riguroso de las sesiones que, en buena parte, correspondieron -como experiencia de práctica y construcción de conocimiento por parte de los profesores investigadores- al enfoque Lesson Study propuesto por Dudley (2014). Por su parte, en la experiencia estuvieron interactuando el profesor de la IED, una docente e investigadora y el investigador autor de este artículo con dinámicas de planeación, observación y análisis (Lewis et. al, 2006) de las actividades y experiencias durante todo el proceso. Esto configuró una comunidad de aprendizaje en procura de mejorar y cualificar los procesos con los estudiantes, tal como lo propone Lim-Ratman (2013).

\section{Análisis y discusión de los resultados}

En el análisis de datos (Figura 1) emergen las categorías a partir de las cuales se estructuró la investigación. Se destaca la distribución homogénea de las categorías hipermedialidad, con 179 citas, y conectividad, con 186, que se consideraron para este estudio como las características potenciadoras de las interacciones que, por vía de la acción pedagógica, permiten la modificación de las circunstancias de los ambientes educativos relacionadas con los aprendizajes, los contenidos, los procesos cognitivos, las dinámicas de participación, las relaciones de los sujetos y los procesos escolares.

La categoría cultura digital aparece con una densidad de 617 unidades de análisis, lo que permitió constituirla como superfamilia. Lo anterior, en virtud de la consideración teórica según la cual la cibercultura o cultura digital es el resultado de la hibridación de los entornos materiales, los simbólicos y los grupos sociales que constituyeron familias dentro de este análisis.

El hecho de que el aspecto pedagógico esté cerca a las 400 unidades de análisis muestra la 


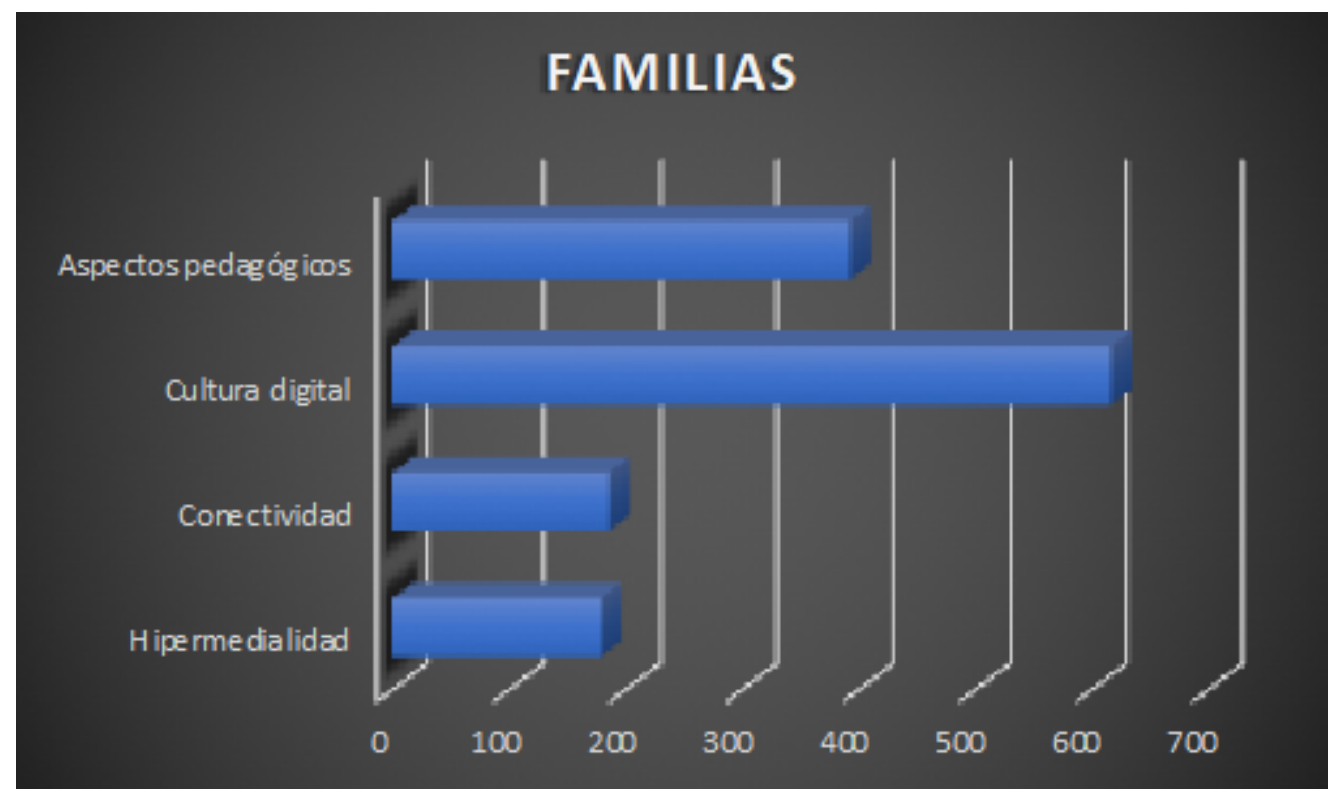

Figura 1. Total de códigos por familia.

Fuente: elaboración propia.

relevancia y pertinencia de este elemento a la luz de las necesarias reflexiones sobre los aportes de este trabajo a la dimensión pedagógica. A continuación, se discriminan los principales hallazgos de la investigación.

\section{El espacio como un hipermedio}

Uno de los hallazgos de este trabajo es que el ambiente estudiado funciona y se configura a la manera de un hipermedio; el hipermedio "salta" de la intangibilidad del software y la pantalla y se instala en la tridimensionalidad del espacio físico en las relaciones entre los sujetos, se apoya en los soportes digitales y se extiende a los diálogos e interacciones de los sujetos a través de las mediaciones de las que dispone el entorno material.

Tal como lo propone Colom (2000), "el contexto físico de la comunicación educativa a su vez comunica a los comunicadores y a los comunicados" (p. 155). El mismo autor, retomando a Tikunov, plantea que la metacomunicación del espacio físico corresponde a tres tipos: el intraindividual, el interindividual y la comunicación grupal.
En este contexto, la disposición del laboratorio de Ático y de la sala de tabletas de la IED aportan una configuración del espacio físico que toma distancia de la tradicional organización en filas -trabajo centrado en la acción del docente y el uso de dispositivos escolares como el cuaderno o el libro de texto- y propone un nuevo "sentido" a la organización y la acción de los estudiantes y profesores en el espacio y su entorno material. A partir de sus decisiones se organizan en grupos pequeños, grandes o de trabajo individual; del uso de las tabletas se deriva la personalización e individualización de la interacción de los estudiantes con tales dispositivos y, en otros momentos, existe un gran potencial para la interacción con los otros, para compartir lo hallado, lo producido, lo vivenciado con el uso de la "tableta gigante" ${ }^{\text {, }}$ que se convierte en otra ventana para la interacción, el compartir, el visibilizar y el concitar la exploración. Se trata, pues, de un hipermedio social, en términos de Colom (2000), generalizado (abierto), grupal, jerarquizado a la vez que distribuido.

9 Refiere al televisor central, mimetizado en el aula, con apariencia de una tableta de 50 pulgadas aproximadamente. 
Las otras formas de intercambio digital no necesariamente implican conexión digital. Se dan por la atracción, por el "me gusta", del contenido visible en la pantalla del que está al lado y que, por efecto del interés y de esa presencia visual o auditiva en las tabletas, genera una especie de contagio por proximidad que permite difundir ubicaciones específicas en la red, aplicaciones, acciones o procesos que resultan atractivos. Surge, pues, un hipermedio social particular (cerrado) interindividual. La tableta se convierte, así, en una órtesis que expande las posibilidades comunicativas tanto en la función como en el contenido. Podríamos entonces afirmar que el lenguaje es asistido $y$ expandido por estos apoyos externos que hacen lo que el propio cuerpo, nuestro lenguaje, nuestras manifestaciones y expresiones no podrían hacer sin ellos.

\section{Hipermedialidad vs. multimodalidad}

La hipermedialidad se caracteriza tanto por la fragmentación de los contenidos como por la interconexión de los nodos que configuran entramados reticulares multilineales, multisecuenciales y multimediales. Esta conexión configura de manera particular los nuevos documentos que no corresponden a la suma de las partes, sino al sentido que tanto los nodos como los enlaces y el contexto les confieren. Así como un dibujo, que en la creación de un cuento de un niño no puede considerarse como un elemento ilustrativo que reitera o complementa lo que el texto dice, sino que, en sí mismo, es portador de intenciones comunicativas y significaciones propias (Kress, 2009). De igual manera, la hipermedialidad no es el encuentro de diversos medios que se enlazan de maneras aleatorias, sino un hiperdocumento en el cual los distintos fragmentos se enlazan de manera intencionada.

Más ubicados en la producción y colaboración de y con hipermedios, Tchernavskij, et. al (2017) se preguntan qué puede aprender el software de la hipermedia, y elaboran algunas reflexiones que dan cuenta de cómo efectivamente las actuales aplicaciones "imponen limitaciones artificiales a la colaboración entre los usuarios, la distribución a través de los dispositivos y los procedimientos cambiantes que ocurren constantemente en el trabajo real" (p. 1). Estas limitaciones se dan en virtud de la arquitectura de encapsulación y separación de programas y datos que resultan opuestos a los principios implicados en la génesis de la hipermedialidad, es decir, la colaboración, la distribución compartida y la flexibilidad de las interacciones. En la experiencia observada, esta limitación de la arquitectura actual de las aplicaciones derivó en la proliferación y las elaboraciones más de tipo multimodal que hipermedial y en los modos de producción de hibridación entre lo digital y lo análogo.

\section{Aspectos pedagógicos}

En esta categoría se destacan: a) el papel del docente, b) la construcción colectiva, c) la relación juego y aprendizaje y d) la condición de la atención al interior de los espacios interconectados e hipermedializados.

\section{a) El papel del docente}

Él ha de estar interesado y ser un explorador de las posibilidades tecnológicas mientras propicia estas mismas dinámicas con sus estudiantes. Así se descentralizan la enseñanza y los aprendizajes: no es exclusivamente el docente el que enseña, tampoco son los estudiantes los únicos que aprenden. El papel del docente se dirige a la identificación de los avances diferenciados de los estudiantes y el favorecimiento de condiciones de trabajo y aprendizaje cooperativo y colaborativo que, tal como lo propuso Vygotsky (1978), acentúan la interacción social como mediación de la construcción de los aprendizajes. Según Panitz (1999), existe una diferencia entre estos dos tipos de aprendizaje que se puede enunciar como el nivel de control sobre los procesos de aprendizaje, en donde es el aprendizaje cooperativo aquel en el que el docente orienta las interacciones y los procesos, y el colaborativo, en el que el control es de los estudiantes. En la experiencia observada hay evidencias de estos dos tipos de aprendizajes, los cuales se encuentran favorecidos por la autonomía o personalización con los dispositivos tecnológicos y por las interacciones surgidas de los intereses y logros diferenciados de los estudiantes. La mediación 
del docente consiste en propiciar andamiajes, scaffolding (Wood, Brunner y Ross, 1976), para quienes tienen alguna dificultad; esto es, apoyarlos, darles pistas. En este contexto, la acción del docente, más que proveer un repertorio de saberes disciplinares, consiste en disponer de estrategias de apoyo: orientación adecuada para el momento, la temática, la dificultad o necesidad expresada por sus estudiantes. Otro de los aspectos de los cuales ha de ocuparse el profesor corresponde a lo que denominaremos la preparación técnica del entorno digital, que suele ser una de sus tareas. Frente a los posibles problemas de conexión se deben usar medios alternativos para la preparación de las tabletas, tales como la conexión por bluetooth o por cable para instalar aplicaciones o material educativo previamente. Finalmente, es papel del docente garantizar que la preparación y puesta en escena escolar incluyan lo hipermedial, lo multimodal y la conectividad de lo que sucederá en el aula.

\section{b) La construcción colectiva de narrativas}

El compartir-se lo que se encuentra, lo que se produce, lo que atrae o llama la atención es una forma no solo de dar a conocer al otro ese objeto de interés, también es darse a conocer en la medida en que lo que se ofrece al otro es parte de lo que soy yo, lo que hago, mis intereses, lo que me gusta, agrada o molesta, lo que considero importante para mí y para los demás. La creación colectiva dinamizada por la orientación y apoyo del docente, que no es una novedad en el aula ${ }^{10}$, ofrece otras alternativas para la creación de historias cooperativamente cuando se emplea la tableta por sus posibilidades multimodales, de intercambio y por el carácter digital de la información, que permiten, entre otras acciones, la edición compartida, copia idéntica de los archivos, combinación con otros elementos digitales, etc. En estas elaboraciones colectivas se reconocen las habilidades propias y de los demás, y se asumen de manera natural papeles diferenciados que por momentos se alternan de acuerdo con los intereses de los participantes y los acuerdos grupales. En este sentido la actividad no es asumida como una tarea, ni siquiera como un proyecto;

10 Rodari (1973) hace propuestas para favorecer la cocreación de la fantasía narrativa. es, en sí misma, un juego en el que se recrea la historia que está en proceso de producción y en la que se participa en un momento como actor, en otro como director, camarógrafo, espectador activo o prosumidor en acción, papeles asumidos por los compañeros en virtud de sus aportes ${ }^{11}$.

\section{c) La relación juego y aprendizaje}

En el trabajo de Jenkins et. al (2003) se muestra la relevancia de asumir la relación juego y educación en atención a distintos factores: el primero de ellos es que, desde hace ya más de una década, se ha identificado que los estudiantes prefieren dedicar buena parte de su tiempo a jugar en los computadores, las consolas, las tabletas y, por supuesto, los teléfonos celulares, antes que ver la televisión, ir al cine o leer libros. El segundo factor tiene que ver con la capacidad tecnológica en los juegos, manifestada en las posibilidades de simulación, el manejo gráfico y de audio, y la flexibilidad y adecuación a los usuarios. El tercer factor está asociado a que muchos de los juegos comerciales, además de entretener, despliegan un gran poder informativo que puede ser potencializado en la actividad escolar.

Durante el desarrollo de la experiencia, tanto en el Centro Ático como en el colegio, no hubo prohibición de la actividad del juego ni del desarrollo de otras actividades como la exploración, descarga y uso de aplicaciones. El interés por los juegos reclama la necesidad del aprendizaje, es una motivación intrínseca, no de aprender -esta es apenas la consecuencia-, pues el interés principal es jugar, pero se hace indispensable saber cómo obtener el juego, apropiar las reglas de los nuevos entornos, reconocer las formas de interacción, los movimientos sobre la pantalla, tomar decisiones en relación con circunstancias dinámicas del juego, etc. Estos aprendizajes, a los cuales subyacen lógicas de interacción, son comunes entre los juegos, otras aplicaciones y el software digital. De esta manera los estudiantes aprenden, en principio, y desarrollan habilidades instrumentales de operación generalizables a diversos tipos de productos

11 Según Toffler (1980), "en vez de clasificar a las personas por lo que poseen, como hace la ética del mercado, la ética del prosumidor atribuye un elevado valor a lo que hacen" (p. 177). 
digitales, mientras que desarrollan actitudes y destrezas exploratorias para la comprensión de los entornos digitales. Esta exploración-apropiación genera procesos de transacción entre los niños que comparten permanentemente aquello que logran y aquello que les interesa. De otra parte, el proyecto de uso de las tabletas permite un alejamiento de las tareas tediosas e indeseables de la escuela ${ }^{12} \mathrm{y}$, si bien varias de las actividades propuestas comportan la necesidad de concentración, producción e ilación de las ideas -actividades no necesariamente sencillas-, es evidente que resultan divertidas e interesantes para los estudiantes.

d) La condición de la atención al interior de los espacios interconectados e hipermedializados

La cultura de lo digital presenta un reto muy importante a considerar dentro del espacio de los ambientes educativos. Se trata de la condición de fragmentación-navegación, de multiestimulaciónmultitarea, de superficialidad (Carr, 2011) y del yo distribuido y conectado (Turkle, 1995; 2012) que hace que la atención se enfrente a estas circunstancias, afectando directamente, tal como lo expone Dussel (2010), los procesos y aspectos pedagógicos de aprendizaje para los cuales resultan de suma importancia la concentración, la profundización, la dedicación, el detenimiento y la permanencia. Para Dussel (2010), la atención se ve "asaltada" por un conjunto de circunstancias, unas de orden pedagógico que descentran la acción de enseñante del profesor hacia lo que los estudiantes hacen para lograr aprendizajes y otras por las circunstancias del entorno tecnológico que "invade" y genera una "batalla" por la atención de los niños, niñas, jóvenes y, por supuesto, de los adultos. Estas circunstancias se amplifican con la incorporación de modelos educativos como el de 1 a 1 de portátiles o tabletas en la escuela, máxime si se dispone de conectividad. Lo que observa Dussel (2010) es que se está frente a un reto en el que las pantallas, con su potencial de descentramiento, generan una comunidad de espectadores radicalmente distinta a la que otrora caracterizara el cara a cara, esto es, conectada en "redes horizontales, con flujos

12 Es importante decir que esto sucedió tanto en el espacio de laboratorio del Ático como en el de la escuela, a pesar de las restricciones técnicas en esta última. imposibles de controlar por el docente y con objetos discretos que habrá que ver cómo se combinan en un relato coherente y en una conversación común" (p. 29).

Dentro de la experiencia observada es evidente que la configuración del espacio, la propuesta de trabajo -que emplea la metodología del trabajo por proyectos- $y$, por supuesto, el uso de las tabletas y la conectividad, alteran la condición de centralidad del docente, de la enseñanza frontal, de la coincidencia de los procesos para todos los estudiantes. En consecuencia, se produce una fragmentación de la atención, una distribución heterogénea de los intereses y acciones de los estudiantes a la vez que de sus aprendizajes.

En suma, hay una atención comúnmente distribuida que suele ser personal y por pequeños grupos. Hay momentos, actividades y estrategias que suscitan la atención compartida de todo el grupo y en los que docentes y estudiantes alternan su centralidad frente al grupo. Corresponde al docente identificar las alternativas que permiten hacer tránsitos entre la atención individualizada, descentrada y fragmentada -que propicia o favorece procesos particulares- hacia momentos en los que la centralidad, la unidad y la homogeneidad avanzan en direcciones deseables del trabajo grupal.

\section{La cultura digital y conectividad}

Como se ha planteado previamente, la cultura digital se describe e interpreta desde tres grandes categorías: a) el entorno simbólico, b) el entorno material y c) el grupo social.

\section{a) El entorno simbólico}

Estos entornos están constituidos por las actividades de carácter simbólico que, por ende, se expresan como representaciones, interpretaciones y percepciones de los sujetos en relación con sus contextos y en sus interacciones sociales. Lo anterior permite integrar aspectos referidos a los universos de sentido que corresponden, tal como lo expone Mignolo (1983), al componente cognoscitivo junto con el componente lingüístico y el pragmático -o contexto de la situación-. Los universos de sentido 
permiten compartir las comprensiones de la cotidianidad que viven los sujetos en determinados momentos, circunstancias y espacios.

En este contexto, resultó pertinente para este estudio identificar cuáles son las representaciones que los sujetos hicieron de las situaciones y espacios vividos durante la experiencia, tanto en el laboratorio como en el aula de la escuela. Las formas diferenciadas en las que se perciben los dos escenarios de interacción tienen que ver con el espacio del laboratorio como símbolo de la novedad, la aventura, la exploración, mientras que el espacio del colegio simboliza lo ya conocido, la obligatoriedad. A esto se suma el hecho de que el Ático es un territorio que simboliza lo tecnológico, la producción; en cambio, la escuela simboliza la reproducción. Esta es una de las ideas reiteradas de los estudiantes, lo que indicaría que la producción en sí misma constituye un elemento clave de la atracción del nuevo territorio: el tecnológico digital.

\section{b) El entorno material}

Dentro de la experiencia resultó evidente no solo la empatía que genera el encuentro con los dispositivos tecnológicos, sino la relación afectiva que se establece con ellos: el gusto, el placer, la complacencia y la motivación fueron expresiones permanentes durante la experiencia observada. Puede afirmarse que los entornos materiales digitales simbolizan y representan objetos de deseo y satisfacción, y que de allí deriva buena parte de su potencial para la educación.

Los entornos materiales, dentro de esta experiencia, se caracterizaron tanto por los usos intensivos de las tabletas, con su potencial interactivo, como por las posibilidades de la conectividad que, entre otras circunstancias, son connaturales al diseño de las tabletas. Al carecer de una memoria o disco duro de gran capacidad, estas últimas dependen, en buena medida, de la red para operar plenamente. Desde el comienzo se privilegió la búsqueda de soluciones por parte de los propios estudiantes antes que la instrucción. Estos procesos de reconocimiento del entorno digital requieren la necesaria dedicación de tiempo a la exploración para resolver un problema o superar un reto. Este tiempo está dedicado a la identificación de posibilidades, muchas de ellas por acierto y error, ya que no necesariamente los estudiantes preguntan a los profesores, pues hay un tiempo de "latencia" o búsqueda propia. Hay un cierto ánimo de autosuficiencia y autonomía incorporado en las acciones y en la solución de problemas dentro de estos entornos que, vía interacción, permiten a los sujetos buscar sus propios caminos de solución.

La idea de que los entornos digitales son ajenos o, por lo menos, distantes a buena parte de los estudiantes -sobre todo los de menores recursos económicos, como los participantes de esta experiencia $^{13}$, o los más pequeños- se desvirtuó, ya que se pudo apreciar que incluso los estudiantes de preescolar poseen un cierto repertorio de saberes que les permiten interactuar, explorar y aprender nuevas cosas con y de los dispositivos. Estos repertorios son el producto de las interacciones previas que los estudiantes han tenido con teléfonos celulares, tabletas o computadores, herramientas que comparten lógicas de operación interactiva que les son comunes. Se puede afirmar que existen unas ciertas improntas culturales relacionadas con los usos y aproximaciones a los entornos artefactuales digitales.

\section{c) El grupo social}

Los estudiantes establecen relaciones individuales y diferenciadas con el entorno artefactual dependiendo no solo de sus intereses personales, sino de los logros, dificultades y satisfacciones que devienen de sus interacciones con los dispositivos y con sus compañeros. Durante la observación se pudo evidenciar el surgimiento, de forma natural, implícita y espontánea, de una cierta regla general: compartir en grupo los nuevos aprendizajes, los productos y las creaciones realizadas por los estudiantes. Este compartir está asociado con expresiones de satisfacción, ya que siempre buscan mostrarlo a los demás al conectar sus tabletas de forma inalámbrica a la "tableta gigante", proceso que hacen con propiedad y en el que también se generan actitudes de respeto por el turno, interés

13 Los estudiantes de la IED son habitantes de los estratos 2 y 3. 
por ver el trabajo del otro, comentarios positivos e incluso aplausos entre ellos. Este reconocimiento social fortalece y propicia superar nuevos retos y compartir los logros. Se podría afirmar que la configuración del entorno material favorece actitudes relacionadas con el compartir-se. Diríamos que ya no solo es la diversificación del yo, sino su exposición, su proyección a través de un recurso que ahora es medio de expresión, representación y exhibición. Se establece así una relación entre lo que se hace y la valoración social que el grupo otorga de lo realizado.

\section{Conclusiones}

En la experiencia observada, el espacio físico se configuró como un hipermedio. Este reunió un conjunto de acciones e interacciones de los actores escolares dentro del ecosistema comunicativo del aula, experimental y de la IED, que permiten comprenderlo como un entorno hipermedializado. En su interior, cada uno de los participantes actúa a la manera de un nodo para el que las tabletas sirven de mediadores, anclas $\mathrm{u}$ órtesis que funcionan como apoyo externo al amplificar las posibilidades de las funciones representacionales y comunicativas de los sujetos: el lenguaje es entonces asistido y expandido. Esto corresponde a una característica de lo que podemos calificar como un rasgo, tal vez incipiente pero evidente, de la inteligencia colectiva en una red. La hipermedialidad en el espacio se manifestó en este estudio intraindividual, interindividual y grupalmente, y corresponde a actividades de navegación o interacción solipsista, compartida con otros y con el grupo general.

De otra parte, se identificaron las potencialidades del juego para propiciar circunstancias que favorecen los aprendizajes, la generación de actitudes para el trabajo colectivo y el desarrollo de habilidades y destrezas técnico-instrumentales. Lo anterior implica considerar dinámicas, tiempos y recursos que le den un lugar en las actividades habituales dentro de las aulas.

Se evidenciaron como una constante los efectos de fragmentación y dispersión de la atención en los estudiantes. Por lo anterior, los docentes han de concebir e implementar el uso de estrategias que convoquen, cuando corresponde, la centralidad de la atención sin desconocer que la personalización y descentramiento individual tiene potencialidades.

Las percepciones de los estudiantes en relación con las representaciones que surgieron en los espacios del laboratorio y del aula de tabletas mostró que se asocia el lugar de la escuela con circunstancias de encierro, restricción y desinterés. El espacio del laboratorio se percibió como un lugar del que se aprende, contrario a la escuela, que parece haber agotado su posibilidad de ser un espacio de aprendizajes: es un lugar en el que se aprende, mas no del que se aprende. He aquí un reto y oportunidad para docentes y arquitectos: la posibilidad de que estos espacios puedan diseñarse y disponerse con semánticas variadas y variables.

Las perspectivas pedagógicas desde las cuales se hacen estas reformulaciones no son tan nuevas como las tecnologías digitales que por su naturaleza conectiva e hipermedial resultan coincidentes con enfoques pedagógicos de larga duración. Es el caso de los planteamientos de la escuela experiencial de Dewey (1960), publicados inicialmente en 1938, los métodos naturales de Freinet (1984), concebidos hacia 1926, los centros de interés de Decroly, también planteados a principios del siglo pasado, el método de proyectos de Kilpatrick, publicado en 1918, entre otros.

La arquitectura de encapsulación y separación de programas y datos de los sistemas, software, hardware y aplicaciones en las tabletas, y que se hace extensiva a los teléfonos inteligentes, no propician ni permiten su integración e interrelación en cuerpos hipermediales. Por lo anterior, lo que prevaleció en este estudio fue la multimodalidad antes que la hipertextualidad.

En la experiencia se pudo evidenciar que la escritura multimodal es favorecida por las posibilidades tecnológicas tanto de integración de lo análogo con lo digital, como por la versatilidad de las tabletas para digitalizar, producir y editar fragmentos de imágenes, videos, audios y textos. 


\section{Referencias}

Bisama, Á. (2002). Hipertextualizar la ciudad letrada. Revista Cyber Humanitatis. Revista de la Facultad de Filosofía y Humanidades. https://web.uchile.cl/ vignette/cyberhumanitatis/CDA/o_2/0,1255,SCID\%2 53D5939\%2526ISID\%253D258,00.html

Bradfield Company, The. (2006). Inanimate Alice (2006-2017) [Narrativa hipertextual]. The Bradfield Company. https://inanimatealice.com/

Carr, N. (2011). Superficiales. ¿Qué está haciendo internet con nuestras mentes?. Taurus.

Clavijo, A., y Quintana, A. (2004). Maestros y estudiantes escritores de hiperhistorias. Universidad Distrital Francisco José de Caldas.

Colom, A. (2000). Metacomunicación y semántica de los espacios educativos. En H. Valderrama (Ed.), Comunicación-Educación. Coordenadas, abordajes y travesías (pp. 155-179). Siglo del Hombre. https:// books.openedition.org/sdh/197

de Corte, E. (1996). Aprendizaje apoyado en el computador: una perspectiva a partir de la investigación acerca del aprendizaje y la instrucción. En G. Barón, O. Mariño y H. Escobar (Coords.), Memorias: III Congreso Iberoamericano de Informática Educativa (Vol. 1) (pp. 40-49). RibielCol.

Dewey, J. (1960). Experiencia y educación. Losada.

Downes, S. (2012). Connectivism and Connective Knowledge. Essays on Meaning and Learning Networks. National Research Council Canada. https://goo.gl/kHSepX

Downes, S. (2019). Recent Work in Connectivism. European Journal of Open, Distance and E-Learning, 22(2), 112-131. https://www.downes.ca/ cgi-bin/page. cgi?post $=69966$

Dudley, P. (Ed.). (2014). Lesson Study: Professional Learning for our Time. Routledge.

Dussel, I. (2010). La educación en la era digital: atención y distracción en aulas conectadas. Todavía. Pensamiento y Cultura en América Latina, 24, 26-31. https://issuu.com/fundacionosde/docs/ todavia-24

Dussel, I., y Gutiérrez, D. (2006). Educar la mirada: políticas y pedagogías de la imagen. Manantial/ Flacso/OSDE.

Dyson, E., Gilder, G., Keyworth, G., \& Toffler, A. (1994). Cyberspace and the American Dream: A Magna Carta for The Knowledge Age. Release 1.2. The information society, 12(3), 295-308.
Echeverría, J. (2001). Indicadores educativos y sociedad de la información. Organización de Estados Iberoamericanos para la Educación, la Ciencia y la Cultura (OEI). https://www.redalyc.org/ pdf/1051/105117890014.pdf

Escobar, A. (2005). Bienvenidos a Cyberia. Notas para una antropología de la cibercultura. Revista de Estudios Sociales, 22, 15-35. https://doi.org/10.7440/ res22.2005.01

Farías, M., y Araya, C. (2014). Alfabetización visual crítica y educación en lengua materna: estrategias metacognitivas en la comprensión lectora de textos multimodales. Colombian Applied Linguistics Journal, 16(1), 93-104. http://dx.doi.org/10.14483/ udistrital.jour.calj.2014.1.a08

Freinet, C. (1984). Los métodos naturales (Vols. I, II, III). Fontanella.

Gardner, H. (1994). Estructuras de la mente. La teoría de las inteligencias múltiples. Fondo de Cultura Económica.

Gibson, W. (1997). Neuromante. Ediciones Minotauro.

González, H., y León, E. (2002). Proyecto ambiente hipermedial para el desarrollo de la didáctica literaria a partir del minicuento (HIMINI). Universidad Pedagógica Nacional.

Gorman, S. (2017). Writing Digital Media. First Draft Syllabus. Provost's Mentored Teaching Fellowship. Samantha Gorman. http://almitras.com/USCFellow syllabus.pdf

Gorman, S., y Cannizzaro, D. (2013). Pry [Narrativa hipertextual]. Tender Claws. http://prynovella.com/

Hamdan, N., Mohamad, M., y Shaharuddin, S. (2017). Hypermedia Reading Materials: Undergraduate Perceptions and Features Affecting their Reading Comprehension. Electronic Journal of e-Learning, 15(2), 116-125. https://files.eric.ed.gov/fulltext/ EJ1141772.pdf

Henao, A. (2002). Procesamiento cognitivo y comprensión de textos en formato hipermedial. Universidad de Antioquia.

Henao, O., Ramírez. L., y Medina, M. (2004). Una propuesta didáctica mediada por recursos informáticos para desarrollar habilidades lectoras en población sorda. En RIBIE (Coord.), Actas: VII congreso iberoamericano de informática educativa (pp. 1021-1030). https://goo.gl/9ruUTH

Hine, Ch. (2004). Etnografía virtual. UOC.

Igarza, R. (2008). Nuevos medios: estrategias de convergencia. La Crujía. 
Jenkins, H., Klopfer, E., Squire, K., y Tan, P. (2003). Entering the Education Arcade. Computers in Entertainment (CIE), 1(1), 1-11. https://dl.acm.org/ doi/pdf/10.1145/950566.950591

Jewitt, C., y Kress, G. (Eds.). (2003). Multimodal Literacy. Lang.

Kilpatrick, W. (1918). The Project Method: The Use of the Purposeful Act in the Educative Process. Teachers College Record, 19(4), 319-335. https://www. tcrecord.org/content.asp? contentid $=3606$

Kress, G. (2000). 'Multimodality' Multiliteracies: Literacy Learning and the Design of Social Futures. En B. Cope y M. Kalantzis (Eds.), Multiliteracies: Literacy Learning and the Design of Social Futures (pp. 182202). Macmillan.

Kress, G. (2003). Literacy in the New Media Age. Routledge.

Kress, G. (2009). Multimodality: A Social Semiotic Approach to Contemporary Communication. Routledge.

Kress, G., Leite-García, R., y van Leeuwen, T. (2000). Semiótica discursiva. En T. van Dijk (Comp.), El discurso como estructura y como proceso. Estudios sobre el discurso I: una investigación multidisciplinaria (pp. 373-416). Gedisa.

Lévy, P. (2004).Inteligenciacolectiva.Poruna antropología del ciberespacio. Organización Panamericana de la Salud. http://inteligenciacolectiva.bvsalud.org/public/ documents/pdf/es/inteligenciaColectiva.pdf

Lévy, P. (2007). Cibercultura. La cultura de la sociedad digital. Anthropos. https://antroporecursos.files. wordpress.com/2009/03/levy-p-1997-cibercultura.pdf

Lewis, C., Perry, R., Hurd, J., y O'Connell, M. (2006). Lesson Study Comes of Age in North America. Phi Delta Kappan, 88(4), 273-281.

Lim-Ratnam, C. (2013). Lesson Study Step by Step: How Teacher Learning Communities Improve Instruction. International Journal for Lesson and Learning Studies, 2(3), 304-306. https://doi. org/10.1108/IJLLS-05-2013-0025

Liu, Ch., Liu, K., Chen, W., Lin, Ch., y Chen, G. (2011). Collaborative Storytelling Experiences in Social Media: Influence of Peer-Assistance Mechanisms. Computers E Education, 57(2), 1544-1556. https:// doi.org/10.1016/j.compedu.2011.02.002

Medina, M. (2003). La cultura de la tecnociencia. En C. Bueno y M. Santos (Coords.), Nuevas tecnologías y cultura (pp. 29-74). Anthropos.

Mignolo, W. (1983). Semiosis y universos de sentido. Lexis, 7(2), 219-237. http://revistas.pucp.edu.pe/ index.php/lexis/article/download/4786/4788
Palumbo, D., y Prater, D. (1993). The Role of Hypermedia in Synthesis Writing. Computers and Composition, 10(2), 59-70. https://www.learntechlib.org/p/146025/

Panitz, T. (1999). Collaborative versus Cooperative Learning: A Comparison of the Two Concepts Which Will Help Us Understand the Underlying Nature of Interactive. ERIC. https://eric.ed.gov/?id=ED448443

Piscitelli, A. (2002). Ciberculturas 2.0: en la era de las máquinas inteligentes. Paidós.

Piscitelli, A., Adaime, I., y Binder, I. (Comps.). (2010). El proyecto Facebook y la posuniversidad. Sistemas operativos sociales $y$ entornos abiertos de aprendizaje. Ariel.

Pohl, M., y Purgathofer, P. (2004). Hypertext Writing Profiles and Visualization. Computers and the Humanities, 38, 83-105. https://doi.org/10.1023/ B:CHUM.0000009226.51168.47

Polit, D., y Hungler, B. (2000). Investigación científica en ciencias de la salud. McGraw Hill.

Pope, J. (2017). Further on Down the Digital Road: Narrative Design and Reading Pleasure in Five New Media Writing Prize Narratives. Convergence: The International Journal of Research into New Media Technologies, 20(10), 1-20. https://doi. org/10.1177/1354856517726603

Quintana, A., Ballesteros, B., y Carreño, A. (1998). El Periódico Electrónico Hipermedial. Una opción tecnológica en la vida escolar. En RIBIE (Coord.), Memorias: IV Congreso Iberoamericano de Informática Educativa (s. p.). Red Iberoamericana de Informática Educativa. http://www.c5.cl/ieinvestiga/ actas/ribie98/123.html

Rama, Á. (1998). La ciudad letrada. Arca.

Rodari, G. (1973). Gramática de la fantasía: introducción al arte de inventar historias. Ediciones del Bronce.

Rodríguez, J. (Ed.). (2011). Narratopedia: reflexiones sobre narrativa digital, creación colectiva $y$ cibercultura. Pontificia Universidad Javeriana. https:// www.javeriana.edu.co/gabriella infinita/principal. htm, http://memoriasycaminos.com/

Rueda, R. (2003). Para una pedagogía del hipertexto: una teoría entre la deconstrucción y la complejidad [Tesis doctoral, Universidad de las Islas Baleares]. Repositorio Institucional Universidad de las Islas Baleares. http:// ibdigital.uib.es/greenstone/collect/tesisUIB/index/ assoc/TDX-1002.dir/TDX-1002103-162409.pdf

Salmerón, L., Naumann, J., García, V., y Fajardo, I. (2017). Scanning and Deep Processing of Information in Hypertext: An Eye Tracking and Cued Retrospective Think-Aloud Study. Journal of Computer Assisted Learning, 33(3), 222-233. https://doi.org/10.1111/ jcal.12152 
Sampieri, R., Collado, C., y Lucio, M. (2010). Metodología de la investigación (5a ed.). McGraw Hill.

Saunders, J. (2013). The Ghosts of Yamaraja [Narrativa hipertextual]. John Saunders. https://elmcip.net/ creative-work/ghosts-yamaraja

Siemens, G. (2004). Conectivismo: una teoría de aprendizaje para la era digital. Semanthics Schoolar. https://pdfs.semanticscholar.org/05f1/ adee187323d66beab226058b23a7416c3517.pdf

Swan, K., y Meskill, C. (1996). Using Hypermedia in Response-Based Literature Classrooms: A Critical Review of Commercial Applications. Journal or Research on Computing Education, 29(2), 167-195. https://doi.org/10.1080/08886504.1996.10782193

Tchernavskij, P., Klokmose, C., y Beaudouin-Lafon, M. (Abril de 2017). What Can Software Learn from Hypermedia?. Programming, 29, 1-5. https://doi. org/10.1145/3079368.3079408

Toffler, A. (1980). La tercera ola. Plaza \& Janes.
Turkle, S. (1995). La vida en la pantalla. La construcción de la identidad en la era de internet (L. Trafi, Trad.). Paidós.

Turkle, S. (2012). Alone Together. Why We Expect More from Technology and Less from Each Other. Basic Books.

Vygotsky, L. (1978). La mente en la sociedad: el desarrollo de las funciones psicológicas superiores. Harvard University Press.

Wilks, Ch. (2010). Underbelly. Christine Wilks. https:// crissxross.net/home

Wood, D., Bruner, J., y Ross, G. (1976). The Role of Tutoring in Problem Solving. Journal of Child Psychology and Psychiatry, 17(2), 89-100. https:// doi.org/10.1111/j.1469-7610.1976.tb00381.x

Yang, S. (1996). A Dynamic reading-linking-to-writing Model for Problem Solving within a Constructive Hypermedia Learning Environment. Journal of Educational Multimedia and Hypermedia, 5(3-4), 283-302. https://dl.acm.org/toc/jemh/1996/5/3-4 